\title{
FILMES DELGADOS LUMINESCENTES OBTIDOS A PARTIR DE HIDROXICARBONATOS DE ÍTRIO ATIVADOS POR EURÓPIO OU TÉRBIO
}

Emy Niyama*,\#, Anselmo Colombo de Alencar, Ligia Delgado da Vila, Elizabeth Berwerth Stucchi e Marian Rosaly Davolos Departamento de Química Geral e Inorgânica, Instituto de Química de Araraquara, Universidade Estadual Paulista, CP 355, 14801-970 Araraquara - SP

Recebido em 24/10/02; aceito em 21/10/03

\begin{abstract}
LUMINESCENT THIN FILMS OBTAINED FROM YTRIUM HYDROXYCARBONATES ACTIVATED BY TERBIUM OR EUROPIUM. These films were obtained by dip coating. Parameters like dislocation velocity; number of deposits, suspension concentration, and number of deposits followed or not by heat treatment between each deposit and calcination temperature were evaluated for establishing the best homogeneity. The obtained films were characterized in terms of their morphology, optical quality and photoluminescence by scanning electron microscopy (SEM), UV-vis absorption spectrophotometry and luminescence spectroscopy, respectively. The morphologic and luminescent characteristics showed dip coating as good laboratory technique for development of thin films for optical applications.
\end{abstract}

Keywords: thin film; dip coating; sol-gel.

\section{INTRODUÇÃO}

A luz proveniente de fonte luminescente origina-se da absorção de outras formas de energia que não sejam de origem térmica. Assim, compostos luminescentes possuem a propriedade de absorver energia quando submetidos a alguma forma de excitação (radiação ultravioleta, raios- $\mathrm{X}$, bombardeamento de elétrons, etc) e convertêla em radiação emitida na forma de fótons. Esta radiação eletromagnética pode ser emitida nas regiões visível, ultravioleta e infravermelho do espectro. Tal fenômeno de conversão de energia eletromagnética é conhecido como luminescência ${ }^{1}$.

As propriedades ópticas dos materiais luminescentes dependem da sua composição e estrutura, mas as características morfológicas ${ }^{2}$ são igualmente importantes para aplicações tecnológicas específicas.

Os materiais luminescentes encontram várias aplicações como fósforos para lâmpadas, em radiologia médica, cintiladores, tubos para raios catódicos, lasers e guias de onda ${ }^{3}$. No setor de visualização de imagens, os materiais eletroluminescentes, processados como filmes finos, têm apresentado um grande interesse para o desenvolvimento de telas planas.

Os filmes podem ser depositados por diversas técnicas, tais como evaporação catódica, pulverização catódica por radiofreqüência e deposição de vapor químico $(\mathrm{CVD})^{5}$. Entretanto, estas apresentam certas limitações quanto ao controle da estequiometria e a extensão das áreas a serem depositadas, além do alto custo operacional. Para contornar tais limitações, técnicas alternativas baseadas em deposições a partir do processo sol-gel ou de suspensões homogêneas podem ser desenvolvidas usando-se técnicas de "dip coating" ou "spin coating" para deposição de filmes finos.

Na técnica de "dip coating", o substrato é emerso de uma suspensão homogênea a uma velocidade controlada. Durante o movimento de emersão a suspensão é arrastada com o substrato, causando um aumento na área de evaporação e na taxa de secagem, for-

\footnotetext{
*e-mail: eniyama@iq.usp.br

\# endereço atual: Instituto de Química, USP, CP 26077, 05513-970 São Paulo - SP
}

mando uma camada delgada. Neste processo, parâmetros como temperatura, velocidade de deposição e viscosidade devem ser controlados, pois determinam a qualidade dos filmes.

A técnica de "dip coating" apresenta uma série de vantagens, tais como: i) obtenção de depósitos sobre substratos com dimensões e formas variadas; ii) razoável controle de espessura; iii) custo operacional baixo; iv) reprodutibilidade dos filmes obtidos; v) quantidades reduzidas de reagentes e vi) controle da estequiometria.

A formação de camadas delgadas pelo processo sol-gel ${ }^{5}$ é influenciada por vários fatores, sendo os mais importantes às características morfológicas do precursor as propriedades físico-químicas da suspensão e as condições de deposição.

Os hidroxicarbonatos precipitados como pós monodispersos através da reação de termólise de uréia ${ }^{6,7}$, podem ser considerados precursores potenciais para filmes delgados, uma vez que se apresentam como partículas homogêneas aproximadamente esféricas e com uma faixa estreita de distribuição granulométrica ${ }^{8}$. Tais características permitem bom empacotamento e possibilitam a utilização de uma quantidade pequena de material polimérico para a deposição das partículas. Além das propriedades morfológicas, os hidroxicarbonatos são termicamente instáveis e podem ser processados a óxidos e oxissulfetos ${ }^{8}$ a temperaturas até $800{ }^{\circ} \mathrm{C}$, resultando em eficientes matrizes luminescentes quando ativados por európio ou térbio. Neste trabalho desenvolveu-se um método de obtenção de filmes luminescentes a partir de hidroxicarbonatos de ítrio dopados com európio ou térbio, depositados através da técnica de "dip coating".

\section{PARTE EXPERIMENTAL}

Os precursores hidroxicarbonatos $\mathrm{Y}_{0,97} \mathrm{~Tb}_{0,03}(\mathrm{OH}) \mathrm{CO}_{3} \cdot \mathrm{H}_{2} \mathrm{O}$ e $\mathrm{Y}_{0,95} \mathrm{Eu}_{0,05}(\mathrm{OH}) \mathrm{CO}_{3} \cdot \mathrm{H}_{2} \mathrm{O}$ foram precipitados através da reação de termólise da uréia com as soluções padronizadas dos respectivos cloretos, conforme descrito em trabalho anterior ${ }^{8}$.

Os filmes foram depositados em substratos de vidro e de quartzo, os quais foram submetidos às temperaturas máximas de $550 \mathrm{e}$ $700{ }^{\circ} \mathrm{C}$, respectivamente. Os depósitos foram realizados a partir de soluções compostas pelo precursor hidroxicarbonato, álcool polivinílico (PVA) e ácido cítrico. 
No preparo das suspensões pesou-se o precursor $(m=0,04 \mathrm{~g})$ e transferiu-se a massa para um béquer de $25 \mathrm{~mL}$, a qual foi suspensa em 24,0 mL de solução saturada de ácido cítrico e 1,0 mL de solução de álcool polivinílico a $10 \%(\mathrm{~m} / \mathrm{m})$. As suspensões preparadas foram aquecidas a $60{ }^{\circ} \mathrm{C}$ por cerca de $30 \mathrm{~min}$.

Os filmes foram depositados sobre substratos previamente lavados com solução "piranha" $\left(3 \mathrm{H}_{2} \mathrm{SO}_{4}: 1 \mathrm{H}_{2} \mathrm{O}_{2}\right)$ (CUIDADO!!! SOLUÇÃO EXTREMAMENTE OXIDANTE, EVITAR CONTATO COM A PELE). Este processo foi realizado em sala climatizada, onde a temperatura foi mantida em torno de $25{ }^{\circ} \mathrm{C}$.

As condições de deposição dos filmes foram obtidas com a variação dos seguintes parâmetros: i) velocidade de deslocamento; ii) número de depósitos; iii) concentração da suspensão; iv) depósitos intercalados com tratamento térmico e v) temperatura de calcinação dos filmes.

Após a deposição dos filmes, estes ficaram fixos no equipamento por 5 min para secagem; posteriormente, foram colocados em estufa por um período de 10 min a $60{ }^{\circ} \mathrm{C}$.

As deposições foram realizadas em duplicata, sendo um dos filmes calcinado à temperatura de $550-700{ }^{\circ} \mathrm{C}$ por um período de $2 \mathrm{~h}$, com rampa de aquecimento de $20^{\circ} \mathrm{C} / \mathrm{min}$.

Os filmes obtidos foram caracterizados quanto à morfologia por Microscopia Eletrônica de Varredura (MEV), quanto à composição química por EDX ("Energy-Dispersive electron probe X-ray analysis") e quanto à qualidade óptica por espectroscopia eletrônica de absorção UV/Vis, usando-se lâminas de quartzo como substratos.

No que se refere às propriedades fotoluminescentes, os filmes foram submetidos à radiação de 200-500 $\mathrm{nm}$ para os espectros de excitação e de 400-800 nm para os espectros de emissão.

\section{RESULTADOS E DISCUSSÃO}

No preparo das suspensões observou-se a liberação de gás $\mathrm{CO}_{2}$ após a adição da solução saturada de ácido cítrico sobre o precursor hidroxicarbonato, o que indica que houve a decomposição deste, mesmo que parcial por tratar-se de ácido fraco, mas o uso do ácido cítrico justifica-se como homogeneizador molecular, para o aumento da aderência do filme ao substrato, além de não gerar ânions residuais na calcinação dos filmes.

Os resultados obtidos através das micrografias indicam uma relação topotática entre o precursor e o produto calcinado formador dos filmes, através da observação de esferas de $\sim 0,1 \mu \mathrm{m}$ de diâmetro - um parâmetro importante para a produção de filmes com distribuição homogênea de partículas.

O filme fino ideal utilizado no setor de visualização de imagens deve apresentar, quanto às características morfológicas, a distribuição uniforme do precursor utilizado, ou seja, a inexistência de aglomerados ou espaços vagos. No que se refere à qualidade óptica, o filme deve ser o mais transparente possível na região visível do espectro para evitar a absorção da luz emitida, fato que implicaria em perda de eficiência da emissão.

Através da caracterização morfológica por MEV pode-se observar a evolução da qualidade dos filmes obtidos, o que se deve às modificações dos parâmetros de deposição utilizados.

O melhor resultado para o estudo da variação do parâmetro de velocidade de deslocamento foi obtido para o filme resultante da velocidade de $50 \mathrm{~mm} / \mathrm{min}$ (Figura 1); assim, tal parâmetro foi fixado para o estudo dos demais parâmetros.

As fotomicrografias dos filmes obtidos com velocidades inferiores a $50 \mathrm{~mm} / \mathrm{min}$ mostram a formação de grandes aglomerados, que implicam em filmes de qualidade inferior, devido à baixa uniformidade.

O segundo parâmetro estudado foi a variação do número de de-

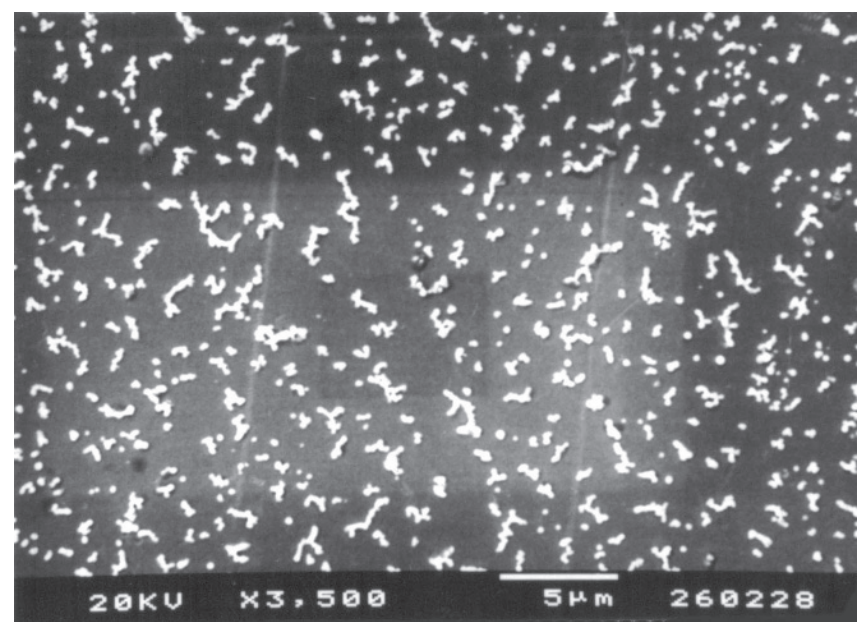

Figura 1. Micrografia do filme de $\mathrm{Y}(\mathrm{OH}) \mathrm{CO}_{3} . \mathrm{H}_{2} \mathrm{O}$, velocidade $=50 \mathrm{~mm} \mathrm{~min}^{-1}$

pósitos. A Figura 2 do filme com 19 depósitos consecutivos apresenta a melhor condição obtida, uma vez que apresentou a distribuição de partículas mais homogênea. As fotomicrografias dos filmes obtidos com menor número de depósitos apresentam cobertura pouco eficiente do substrato e os filmes com maior número de depósitos apresentam a formação de aglomerados.

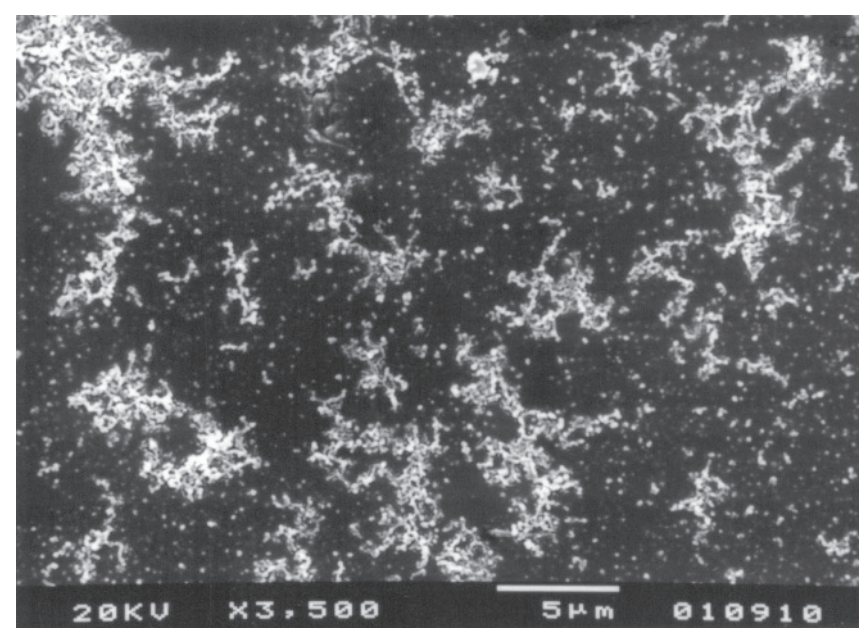

Figura 2. Micrografia do filme de $\mathrm{Y}(\mathrm{OH}) \mathrm{CO}_{3} \cdot \mathrm{H}_{2} \mathrm{O}$ com 19 depósitos

A variação da concentração foi o terceiro parâmetro variado, aumentando-se de $8,6 \mathrm{mmol} \mathrm{L}^{-1}$ para $43 \mathrm{mmol} \mathrm{L}^{-1}$. O filme obtido apresenta a formação de grandes aglomerados e uma cobertura ineficiente da superfície do substrato (Figura 3).

A realização de depósitos intercalados com tratamento térmico a $550{ }^{\circ} \mathrm{C}$ foi o quarto parâmetro estudado. A Figura $4 \mathrm{a}$, correspondente ao filme com 3 depósitos intercalados, mostra um resultado similar quanto à área depositada quando comparado ao filme de 19 depósitos consecutivos. Menor quantidade de aglomerados é observada para o filme intercalado, o que se deve à realização do tratamento térmico após cada deposição.

O tratamento térmico permite calcinar a matéria orgânica dos filmes depositados, o que impede que a nova camada depositada incorpore os aglomerados eventualmente formados, possibilitando a cobertura mais eficiente do substrato. Na Figura $4 \mathrm{~b}$ observa-se o efeito do aumento da temperatura de tratamento térmico, que indica a homogeneidade do filme obtido a $700{ }^{\circ} \mathrm{C}$, evidenciando a importância da temperatura de tratamento térmico. 


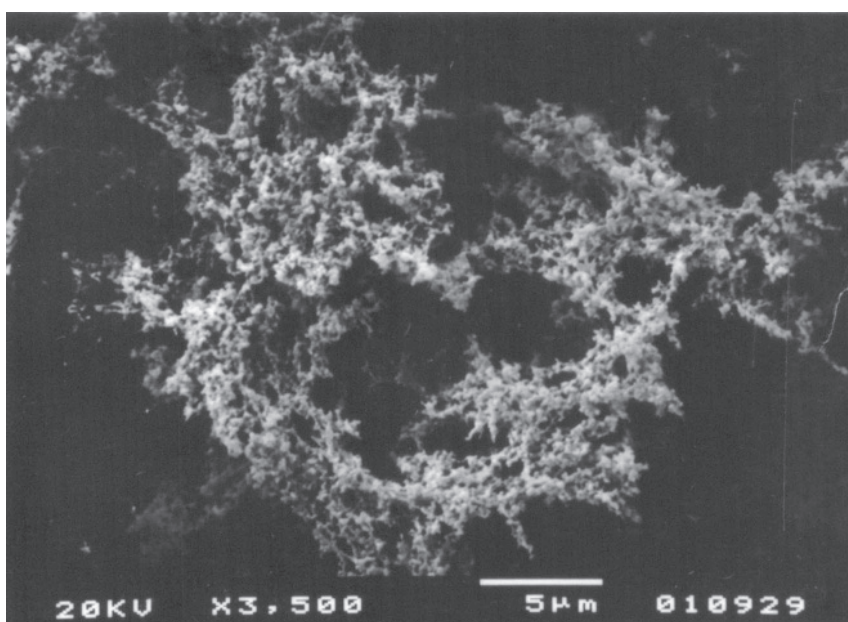

Figura 3. Micrografia do filme de $\mathrm{Y}(\mathrm{OH}) \mathrm{CO}_{3} \cdot \mathrm{H}_{2} \mathrm{O}$ de concentração $43 \mathrm{mmol} \mathrm{L}$
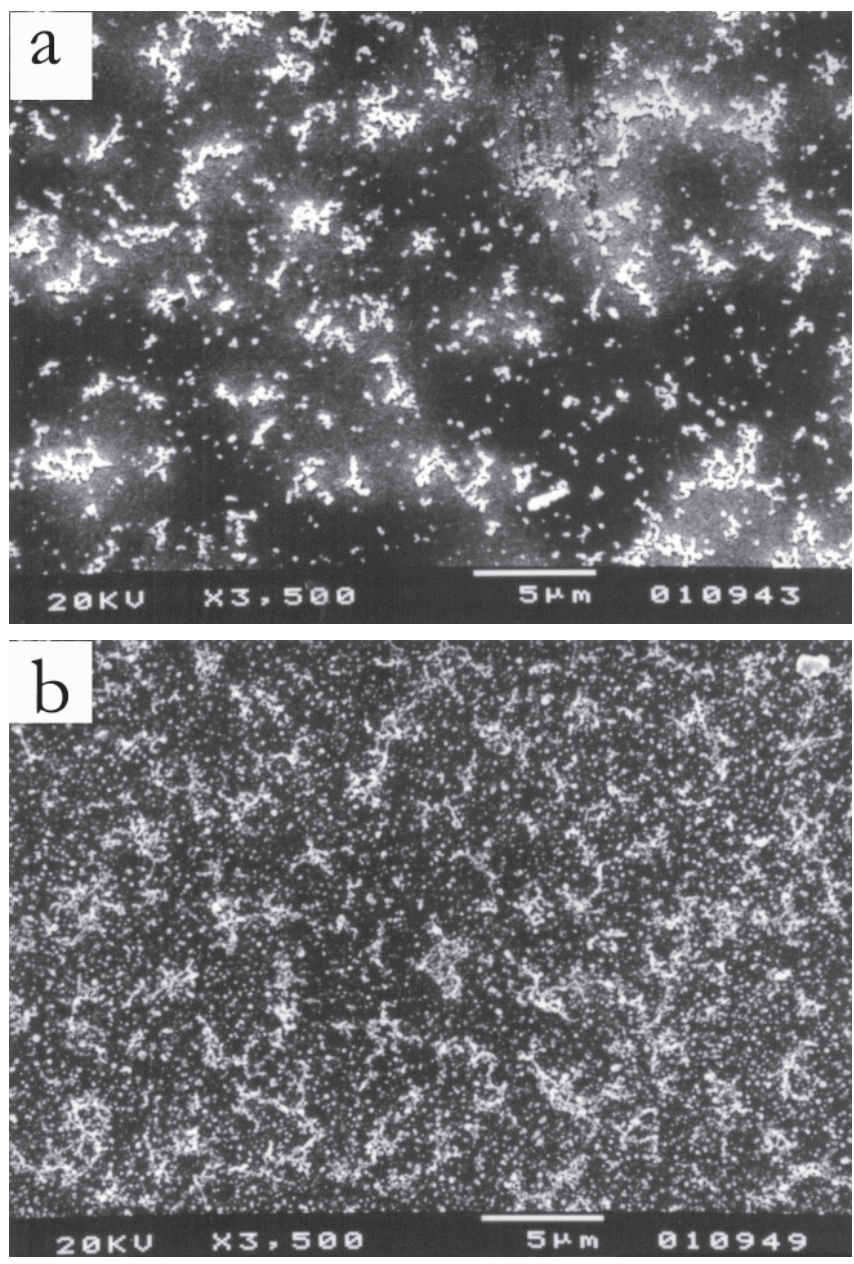

Figura 4. Micrografias do filme de $\mathrm{Y}(\mathrm{OH}) \mathrm{CO}_{3} \mathrm{H}_{2} \mathrm{O}$ : a) 3 depósitos intercalados com tratamento térmico a $550{ }^{\circ} \mathrm{Ce}$ b) 2 depósitos da suspensão de 8,6 mmol L $\mathrm{L}^{-1}$ a $700{ }^{\circ} \mathrm{C}$

Determinados os melhores parâmetros, estes foram utilizados para deposição dos filmes a partir de precursores dopados. Nas Figuras 5 a e 5 b observam-se, respectivamente, os filmes contendo térbio (III) e európio (III) como ativador.

$O$ estudo da uniformidade do dopante terra rara nos filmes foi realizado por EDX; a fotomicrografia obtida (Figura 6) indica a dis-
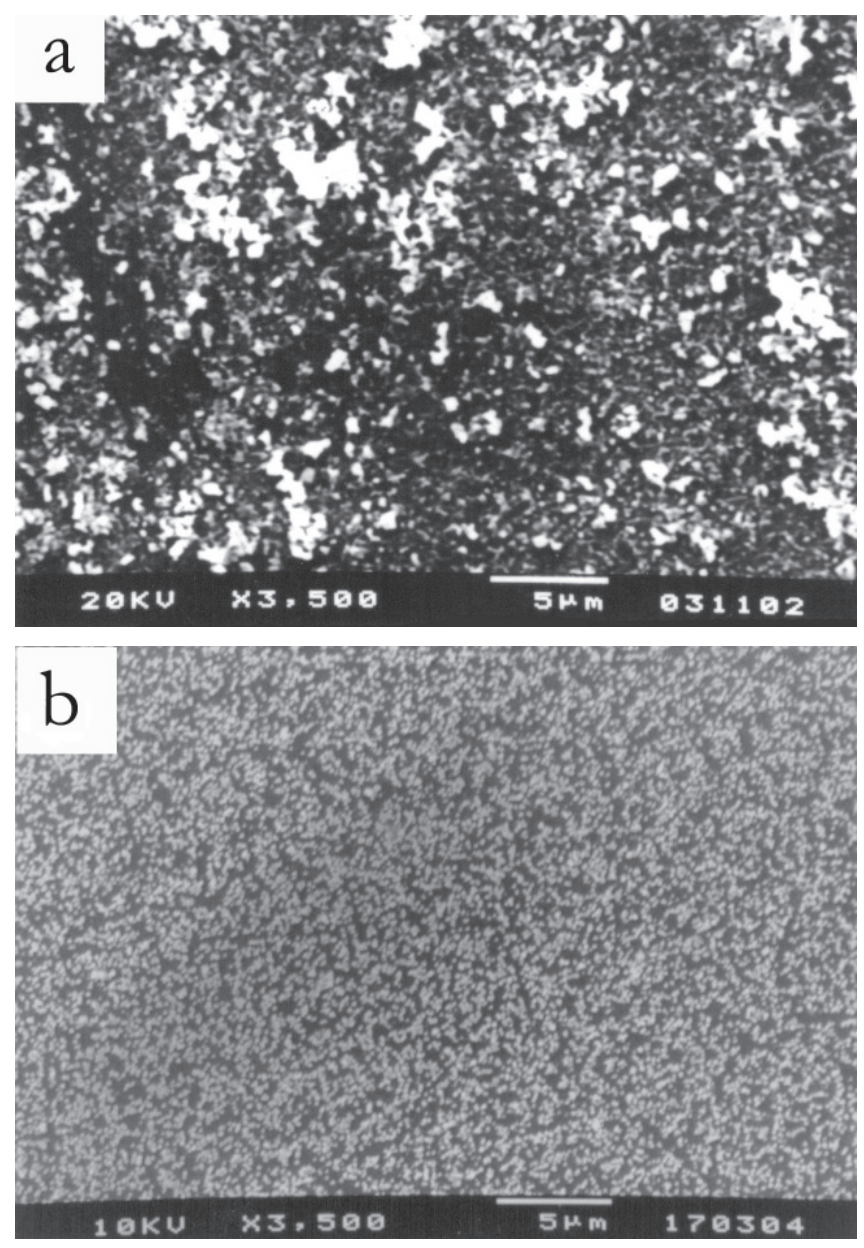

Figura 5. Micrografia dos filmes: a) 10 depósitos obtidos da suspensão de $Y_{0_{97}} \mathrm{~Tb}_{\mathrm{OO}_{3}}(\mathrm{OH}) \mathrm{CO}_{3} \cdot \mathrm{H}_{2} \mathrm{O}$ com concentração de $17,4 \mathrm{mmol} \mathrm{L}^{-1}$ a $700{ }^{\circ} \mathrm{C} e$ b) 2 depósitos obtidos da suspensão de $\mathrm{Y}_{0,95} \mathrm{Eu}_{0,05}(\mathrm{OH}) \mathrm{CO}_{3} . \mathrm{H}_{2} \mathrm{O} \mathrm{com}$ concentração de $8,6 \mathrm{mmol} \mathrm{L}^{-1}$ a $700{ }^{\circ} \mathrm{C}$

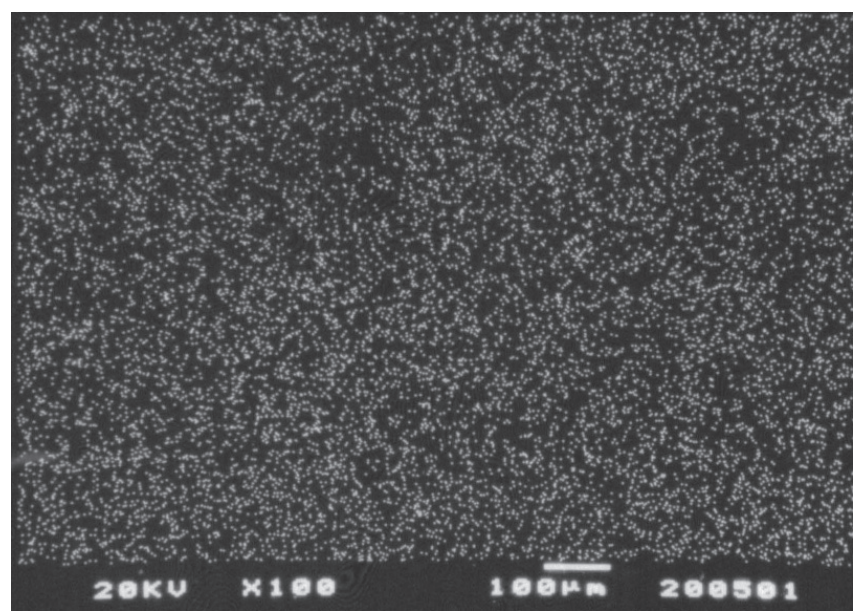

Figura 6. Micrografia (EDX) do filme de 4 depósitos de $\mathrm{Y}_{0,97} \mathrm{~Tb}_{0,03}(\mathrm{OH}) \mathrm{CO}_{3}$. $\mathrm{H}_{2} \mathrm{O}$ depositado em substrato de quartzo

tribuição homogênea do íon térbio no filme de $\mathrm{Y}_{0,97} \mathrm{~Tb}_{0,03}(\mathrm{OH}) \mathrm{CO}_{3} \cdot \mathrm{H}_{2} \mathrm{O}$ com 10 depósitos.

A qualidade óptica dos filmes foi determinada por espectroscopia eletrônica de absorção na região do UV/Vis. Os espectros de transmitância obtidos referentes aos filmes da matriz $\mathrm{Y}(\mathrm{OH}) \mathrm{CO}_{3} \cdot \mathrm{H}_{2} \mathrm{O}$ contendo um depósito (Figura 7 - curva A) e ao filme ativado por Tb 
(III) contendo 4 depósitos (Figura 7 - curva B) apresentam absorção significativa até $400 \mathrm{~nm}$, atribuída à absorção da matriz. Entretanto na região de 400 a $800 \mathrm{~nm}$, ou seja, na região do visível que é útil para emissão, os valores de transmitância encontram-se entre 71$88 \%$ para o filme ativado por $\mathrm{Tb}^{3+}$.

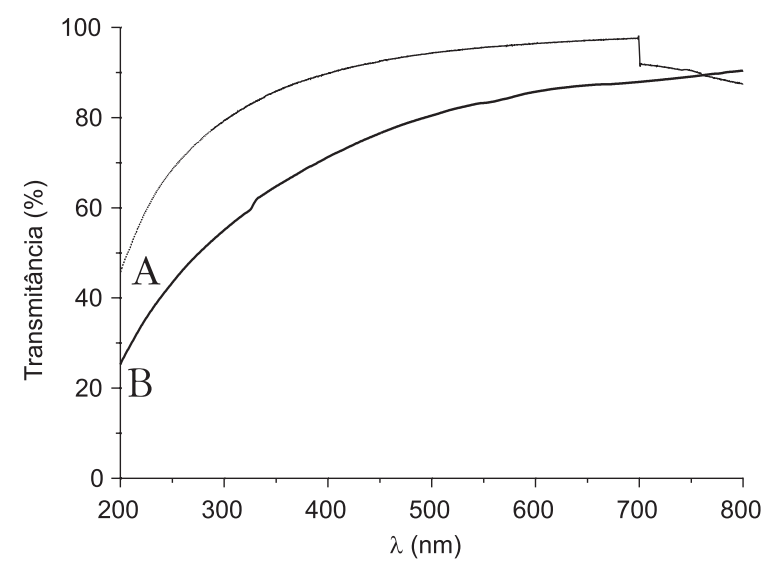

Figura 7. Espectros de transmitância dos filmes depositados em substrato de quartzo: curva A - filme com 1 depósito de $\mathrm{Y}(\mathrm{OH}) \mathrm{CO}_{3} . \mathrm{H}_{2} \mathrm{O}$ e curva $\mathrm{B}$ filme com 4 depósitos de $\mathrm{Y}_{0,97} \mathrm{~Tb}_{0,03}(\mathrm{OH}) \mathrm{CO}_{3} . \mathrm{H}_{2} \mathrm{O}$

Os espectros de luminescência foram obtidos para os filmes ativados por európio e térbio contendo, respectivamente, 30 e 10 depósitos. Além disso, foram obtidos espectros de luminescência do filme de $\mathrm{Y}(\mathrm{OH}) \mathrm{CO}_{3} \cdot \mathrm{H}_{2} \mathrm{O}$, com 1 depósito para o estudo das propriedades fotoluminescentes da matriz.

No espectro de excitação para o filme ativado por európio (Figura 8a), observa-se uma banda larga com máximo a $270 \mathrm{~nm}$, que pode ser atribuída à absorção da matriz e bandas estreitas referentes à transição $f \rightarrow f$ do európio. Dentre estas, a mais intensa é aquela localizada à $394 \mathrm{~nm}$ devido à transição ${ }^{7} \mathrm{~F}_{0} \rightarrow{ }^{5} \mathrm{~L}_{6}$.

Na Figura 8b (curvas A e B), os espectros de emissão do mesmo filme, sob excitação em diferentes comprimentos de onda, sugerem a ocorrência de mais de um sítio de simetria. Na comparação com os espectros do precursor hidroxicarbonato de ítrio dopado com európio (Figuras 9a e 9b), esta hipótese é reforçada.

Para o filme ativado com térbio, a análise espectral é mais complexa devido ao seu estado emissor degenerado ${ }^{5} \mathrm{D}_{4}$, o qual pode se desdobrar em nove componentes, e prejudicada pela baixa intensidade das linhas, causada pelo menor percentual de dopagem em relação ao composto de európio.

No espectro de excitação (Figura 10a - curva A), observa-se que as bandas referentes às transições $f \rightarrow \mathrm{d}$ e $f \rightarrow f$ do térbio estão sobrepostas às bandas largas, uma vez que a linha base do espectro é elevada. As bandas largas poderiam ser atribuídas à presença de radicais ou à participação de absorção da matriz, fato que pode ser constatado através da análise do espectro de excitação do filme da matriz de $\mathrm{Y}(\mathrm{OH}) \mathrm{CO}_{3} \cdot \mathrm{H}_{2} \mathrm{O}$ (Figura 10a - curva B), pois se pode observar a presença de bandas largas.

A presença de radicais torna-se mais evidente quando o espectro de excitação é monitorado com comprimento de onda de emissão em $430 \mathrm{~nm}$ (Figura 10b - curva A) e comprovada através do estudo do espectro de excitação do filme da matriz $\mathrm{Y}(\mathrm{OH}) \mathrm{CO}_{3} \cdot \mathrm{H}_{2} \mathrm{O}$ (Figura $10 \mathrm{~b}$ - curva B). Desta forma, pode-se comprovar que a banda observada refere-se à matriz.

Quando se analisa o espectro de emissão da Figura 11 (curvas A e B) nota-se a ocorrência de uma banda larga na região do azul, cujas características não correspondem à emissão do ativador.
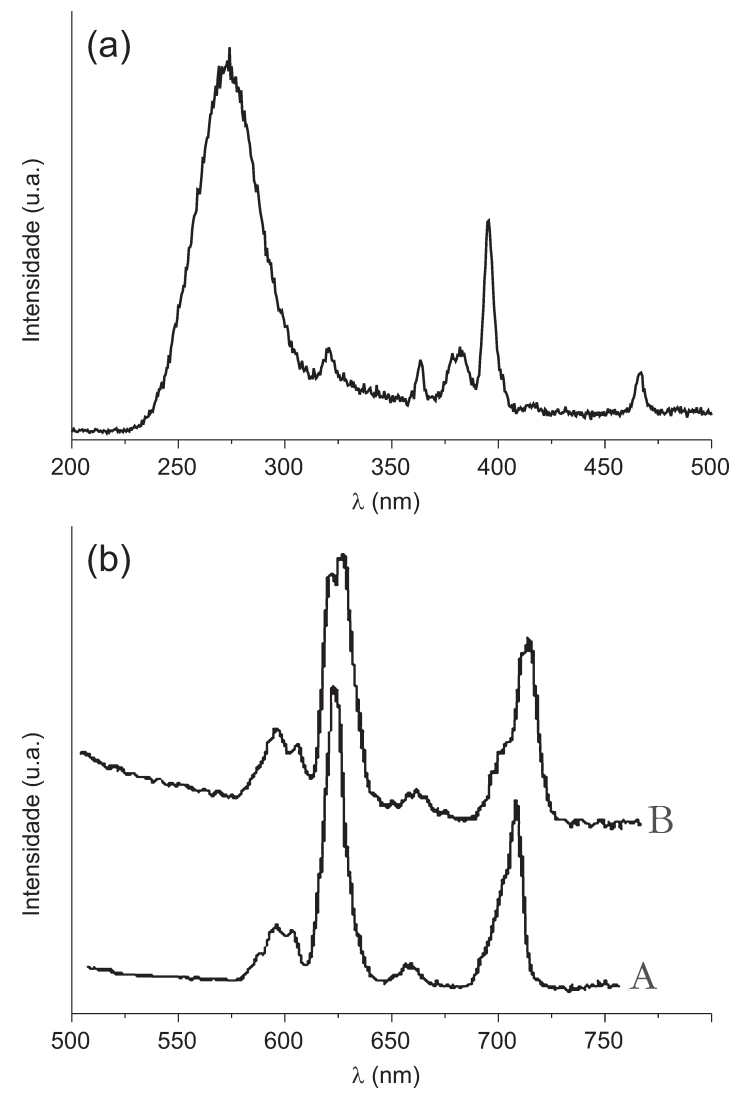

Figura 8. Espectros do filme de 30 depósitos de $\mathrm{Y}_{0,95} \mathrm{Eu}_{0,05}(\mathrm{OH}) \mathrm{CO}_{3} \cdot \mathrm{H}_{2} \mathrm{O}:$ a) excitação $-\lambda_{\text {em }}=616 \mathrm{~nm}$ e b) emissão: curva A) $\lambda_{\text {exc }}=270 \mathrm{~nm}$ e curva $\left.B\right) \lambda_{\text {exc }}$ $=394 \mathrm{~nm}$
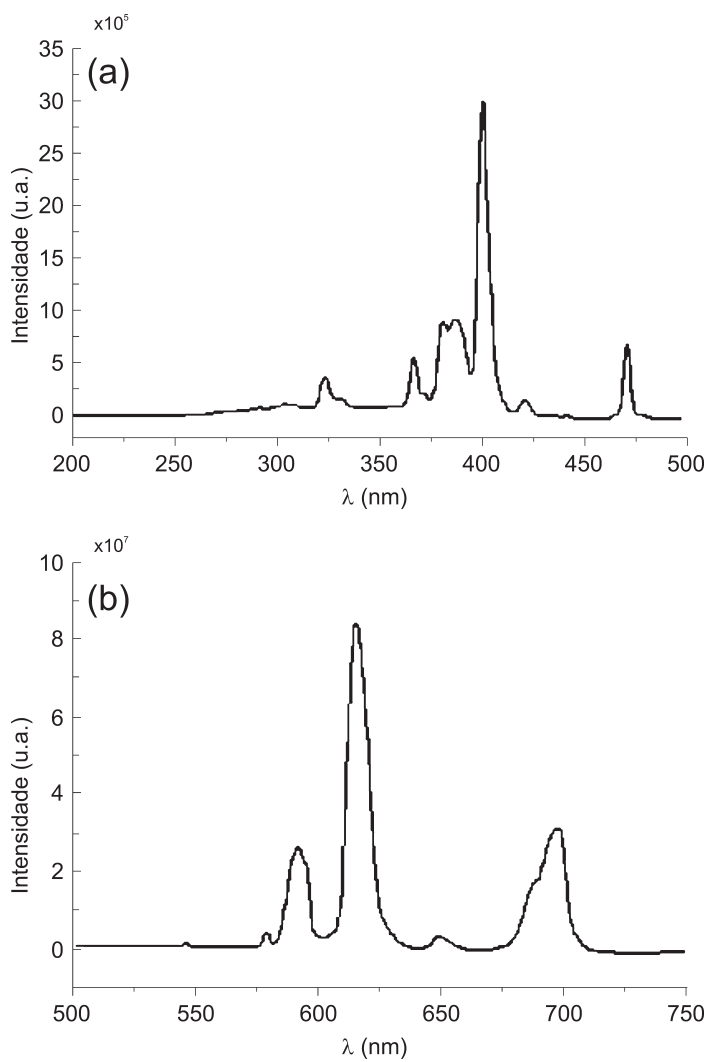

Figura 9. Espectros de $\mathrm{Y}_{0,95} \mathrm{Eu}_{0,05}(\mathrm{OH}) \mathrm{CO}_{3} \cdot \mathrm{H}_{2} \mathrm{O}:$ a) excitação $-\lambda_{e m}=616 \mathrm{~nm}$ e b) emissão $-\lambda_{\text {exc }}=394 \mathrm{~nm}$ 


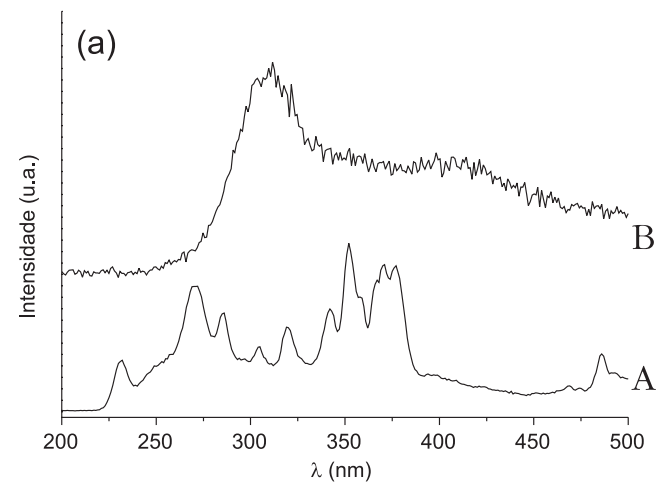

(b)

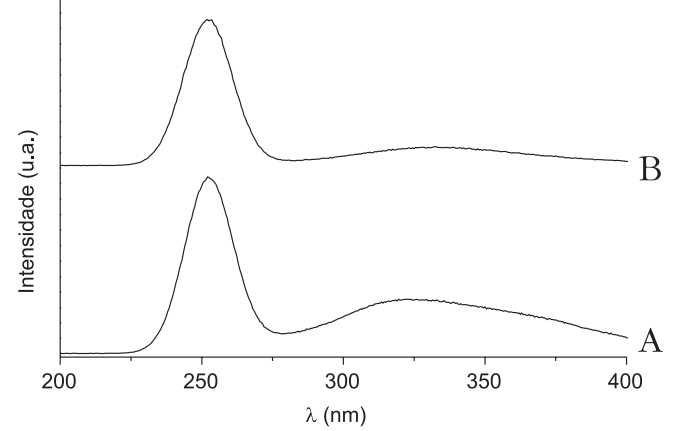

Figura 10. a) Espectro de excitação do filme, $\lambda_{e m}=542 \mathrm{~nm}$ : curva A) 10 depósitos de $\mathrm{Y}_{0.97} \mathrm{~Tb}_{0.03}(\mathrm{OH}) \mathrm{CO}_{3} \cdot \mathrm{H}_{2} \mathrm{O}$ e curva B) 1 depósito de $\mathrm{Y}(\mathrm{OH}) \mathrm{CO}_{3} \cdot \mathrm{H}_{2} \mathrm{O}$; b) espectro de excitação do filme, $\lambda_{e m}=430 \mathrm{~nm}$ : curva A) 10 depósitos de $\mathrm{Y}_{0,97} \mathrm{~Tb}_{0,03}(\mathrm{OH}) \mathrm{CO}_{3} \cdot \mathrm{H}_{2} \mathrm{O}$ e curva B) 1 depósito de $\mathrm{Y}(\mathrm{OH}) \mathrm{CO}_{3} \cdot \mathrm{H}_{2} \mathrm{O}$

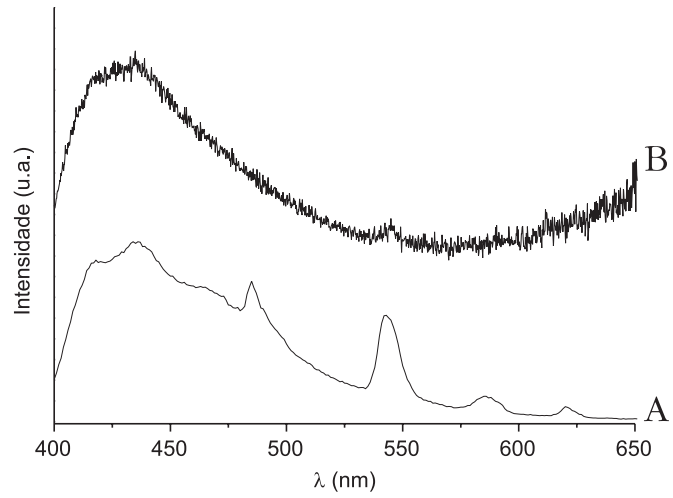

Figura 11. Espectro de emissão dos filmes com $\lambda_{\text {exc }}=370 \mathrm{~nm}$ : curva A) filme de 10 depósitos de $\mathrm{Y}_{0,97} \mathrm{~Tb}_{0,03}(\mathrm{OH}) \mathrm{CO}_{3} \cdot \mathrm{H}_{2} \mathrm{O}$ e curva B) filme de 1 depósito de $\mathrm{Y}(\mathrm{OH}) \mathrm{CO}_{3} \cdot \mathrm{H}_{2} \mathrm{O}$
A participação de radicais $\mathrm{CO}_{2}$ nos processos de luminescência já foi observada por Sigoli e Davolos ${ }^{9}$ em produtos de calcinação de oxalatos sob atmosfera redutora. Provavelmente há formação dos radicais $\mathrm{CO}_{2}$ durante os tratamentos térmicos dos filmes, o que é evidenciado pela emissão observada nos filmes de $\mathrm{Y}(\mathrm{OH}) \mathrm{CO}_{3} \cdot \mathrm{H}_{2} \mathrm{O}$ e de $\mathrm{Y}_{0,97} \mathrm{~Tb}_{0,03}(\mathrm{OH}) \mathrm{CO}_{3} \cdot \mathrm{H}_{2} \mathrm{O}$.

\section{CONCLUSÕES}

Os resultados obtidos das diversas técnicas de caracterização permitem concluir que: i) as melhores cobertura e distribuição de partículas são obtidas quando os depósitos são efetuados sobre camada termicamente tratada; ii) os filmes apresentam alta transparência na região do visível, com transmitância variando de $95 \%$ para bicamadas do filme ativado por európio, e $80 \%$ para tetracamada do filme ativado por térbio; iii) os filmes ativados apresentam fotoluminescência sendo excitados, preferencialmente, através de espécies constituintes ou presentes no retículo hospedeiro, com possível ocorrência de mais de um sítio de simetria; iv) a técnica de "dip coating" e o uso de hidroxicarbonato como precursor em meio de ácido cítrico constituem-se em uma rota rápida e barata para o desenvolvimento de filmes delgados para aplicações ópticas.

\section{REFERÊNCIAS}

1. Blasse, G.: Grabmaier, B. C.; Luminescent Materials, Springer-Verlag: Berlin, 1994.

2. Blasse, G.; J. Alloys Compd. 1995, 225, 529.

3. Ronda, C. R.; Jüstel, J.; Nikol, H.; J. Alloys Compd. 1998, 277, 669.

4. Leskelä, M.; J. Alloys Compd. 1998, 277, 702.

5. Brinker, C. J.; Scherer, G. W.; Sol-gel Science the Physical and Chemistry of Processing, Academic Press: Boston, 1990.

6. Akinc, M.; Sordelet, D.; Adv. Ceram. Mater. 1987, 2, 232.

7. Sordelet, D.; Akinc, M.; J. Colloid Interface Sci. 1988, 122, 47.

8. Vila, L. D.; Stucchi, E. B.; Davolos, M. R.; J. Mater. Chem. 1997, 7, 2113.

9. Sigoli, F. A.; Davolos, M. R.; Jafelicci, M.; J. Alloys Compd. 2002, 344, 308. 